\title{
Evaluation of AAV-Mediated Gene Therapy with Reduced Vector Volume in Cngb3 Knockout Mice, a Model of Achromatopsia
}

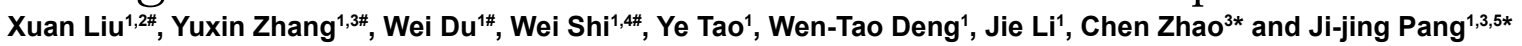

${ }^{1}$ Department of Ophthalmology, College of Medicine, University of Florida, Gainesville, Florida

${ }^{2}$ Tsinghua University Beijing Tsinghua Changgung Hospital, Beijing, China;

${ }^{3}$ Department of Ophthalmology, First Affiliated Hospital, Nanjing Medical University, Nanjing, Jiangsu, China

${ }^{4}$ Beijing Children's Hospital, Capital Medical University, Beijing, China

${ }^{5}$ School of Ophthalmology and Optometry, Eye Hospital, Wenzhou Medical University, Wenzhou, Zhejiang, China

*Contributed equally to this study.

\begin{abstract}
Purpose: This study was designed to investigate whether the volume of vector used for subretinal injection can be reduced to transfect $\mathrm{C} 57 \mathrm{bl} / 6 \mathrm{~J}$ mouse whole retina and whether it can restore cone function in a Cngb3 knockout (KO) mouse model.
\end{abstract}

Methods: C57bl/6J mice and Cngb3 KO mice received a subretinal injection of $0.5 \mu \mathrm{L}$ or $1 \mu \mathrm{L}$ of AAV5-smCBAmCherry vector and AAV5-IRBP/GNAT2-hCngb3 vector, respectively. Retinal whole mounts and frozen sections were prepared from the wild-type mouse eyes to evaluate the transfected area. Dark and light-adapted electroretinograms (ERGs) were recorded two months after vector injection in the eyes of Cngb3 KO mice.

Result: In the retina of AAV5-smCBA-mCherry injected wild-type mice, no difference was observed between the injection volumes. mCherry positive retinal pigment epithelial (RPE) and photoreceptor cells were observed throughout the entire retina. In AAV5-IRBP/GNAT2-hCngb3-injected Cngb3 KO mice, 1- $\mu$ L-injected mice showed a higher average of photopic ERG restoration than $0.5-\mu \mathrm{L}$-injected mice. However, the scotopic ERGs were lower in $1-\mu \mathrm{L}-i n j e c t e d$ mice, indicating that higher injection volumes resulted in more damages.

Conclusion: Reduced volume $(0.5 \mu \mathrm{L})$ of vector induced fewer damages. However, higher doses of vector $(1 \mu \mathrm{L})$ restore higher ERG function in Cngb3 KO mouse.

Keywords: Cngb3KO mice; Subretinal injection; AAV volume; Injection-related damage; Functional rescue

\section{Introduction}

Inherited retinal diseases affect more than 200,000 Americans and millions of individual worldwide [1,2]. Most of them lost their sight gradually during their life. Over the past few decades, more than 200 genes have been identified as the causes of inherited retinal diseases [3,4]. With this knowledge, progress of gene-replacement therapies for several inherited retinal diseases has been made through pre-clinical and clinical studies in recent years [5-10].

Trans-cornea subretinal injection is the most common drug delivery method of gene therapy vectors in mouse models of inherited retinal diseases. The entire retina can be transfected with one shot to exclude the possibility that the untreated degenerating retina releases some toxic materials and influences the treated area in the long term. This technique ensures vector direct contact with retinal pigment epithelial (RPE) and photoreceptor cells. Clinical trials proved that the retinal area responding to therapy is limited to the bleb area detached by the injection [9]. On the other hand, subretinal injection-induced retinal detachment harms the photoreceptors, although the blebs formed by subretinal injection usually regress within $24 \mathrm{hrs}$ [11]. Jacobson et al., reported that subretinal injection under the fovea resulted in decreased visual acuity and fovea thinning in both Leber congenital amaurosis (LCA) 2 patients and nonhuman primates [12-14]. Thus, balancing the rescue effect, while reducing damages caused by subretinal injection, is essential. In this study, we first evaluated whether using a reduced volume of vector allows the transfection of the entire retina in wild type mice and then evaluated if reducing the injection volume can minimize the injection-related damage, while retaining its rescue effect in the Cngb3 knockout $(\mathrm{KO})$ mouse model, the most common model of achromatopsia (ACHM).

\section{Methods}

\section{Animals}

C57 BL/6J mice and Cngb3 knockout (KO) mice were purchased from Jackson Laboratory (Bar Harbor, ME, USA). All mice were maintained in the University of Florida Health Science Center Animal Care Services Facilities under a $12 \mathrm{hr}$ light/dark cycle condition. Cage illumination was approximately 7 foot-candles during the light cycle. All the experiments were approved by the local institutional animal care and use committee and conducted in accordance with the ARVO statement for the use of animals in ophthalmic and vision research.

\section{AAV5 vectors and subretinal injection}

The recombinant adeno-associated virus construct with AAV2 inverted terminal repeats and pseudo-typed AAV5 capsid was based on the pTR-UF2 vector [15]. Serotype 5 rAAV vectors carrying a human Cngb3 cDNA (AAV5-IRBP/GNAT2-hCngb3) and mCherry (AAV5smCBA-mcherry) were prepared as described previously [16]. AAV5smCBA-mcherry and AAV5-IRBP/GNAT2-hCngb3 viral preparations were adjusted to physical particle titers of $10 \mathrm{e} 10 \mathrm{vg} / \mu \mathrm{L}$.

For subretinal injection, $10 \mathrm{P} 14 \mathrm{C} 57 \mathrm{bl} / 6 \mathrm{~J}$ mice were given 1\% atropine eye drops $1 \mathrm{hr}$ before anesthesia using ketamine $(72 \mathrm{mg} /$

*Corresponding author: Ji-jing Pang, Department of Ophthalmology, College of Medicine, University of Florida, Gainesville, Florida, USA, Tel: 352-273-9341; E-mail: jpang@ufl.edu

Chen Zhao, Department of Ophthalmology, First Affiliated Hospital Nanjing Medical University, Nanjing, Jiangsu, China, Tel: 86-25-68135357, E-mail:dr_zhaochen@163.com

Received November 24, 2015; Accepted February 23, 2016; Published February 26, 2016

Citation: Liu X, Zhang Y, Du W, Shi W, Tao Y, et al. (2016) Evaluation of AAVMediated Gene Therapy with Reduced Vector Volume in Cngb3 Knockout Mice, a Model of Achromatopsia. Hereditary Genet 5: 163. doi:10.4172/21611041.1000163

Copyright: (c) 2016 Liu X, et al. This is an open-access article distributed under the terms of the Creative Commons Attribution License, which permits unrestricted use, distribution, and reproduction in any medium, provided the original author and source are credited. 
$\mathrm{kg}) /$ xylazine $(4 \mathrm{mg} / \mathrm{kg})$ intraperitoneal injection, followed by topical administration of $2.5 \%$ phenylephrine hydrochloride eye drops. An aperture within the pupil area was made through the superior cornea with a 30 G-gauge disposal needle. A 33-gauge unbeveled blunt needle mounted on a $5 \mu \mathrm{L}$ Hamilton syringe (Hamilton Co., Reno, NV, USA) was then introduced through the corneal opening, avoiding the lens and penetrating to reach the subretinal space. AAV5-smCBA-mcherry vector $(0.5 \mu \mathrm{L}$ and $1 \mu \mathrm{L})$ was slowly injected into the subretinal space of both eyes $(0.5 \mu \mathrm{L}$ into the right eye and $1 \mu \mathrm{L}$ into the left eye). Two weeks later, 8 mice were euthanized for whole mount preparation and two mice were euthanized for frozen section preparation. The injected retinal area was visualized by fluorescein-positive subretinal blebs [17]. Cngb3 KO mice at P14 were subretinally injected with AAV5-IRBP/ GNAT2-hCngb3 in both eyes, $0.5 \mu \mathrm{L}$ into the right eye and $1 \mu \mathrm{L}$ into the left eye, as described above.

\section{Electroretinogram (ERG) analysis}

After at least $6 \mathrm{~h}$ of adaptation to the dark, mice were anesthetized with an intraperitoneal injection of normal saline solution containing ketamine $(75 \mathrm{mg} / \mathrm{kg}) / x y l a z i n e ~(4 \mathrm{mg} / \mathrm{kg}$ body weight). ERGs were recorded after pupil dilation ( $1 \%$ atropine sulfate) using a gold loop electrode referenced to a gold wire in the mouth. A needle electrode placed in the tail served as ground. A drop of methylcellulose $(2.5 \%)$ was placed on the corneal surface to ensure electrical contact and to maintain corneal integrity. Body temperature was maintained at a constant temperature of $38^{\circ} \mathrm{C}$ using a heated water pad. All stimuli were presented in a Ganzfeld dome (LKC Technologies, Gaithersburg, MD, USA with the interior surface painted with a highly reflective white matte paint (No. 6080; Eastman Kodak, Rochester, NY, USA). Stimuli were generated with a Grass photic stimulator (model PS33 plus; Grass instruments, Worcester, MA, USA) affixed to the outside of the dome at $90^{\circ}$ of the viewing porthole.

Dark-adapted-ERGs were assessed at a stimulus intensity of $0.4 \mathrm{log}$ cd-s $/ \mathrm{m}^{2}$ and inter-stimulus intervals of 30 seconds, with 10 recordings averaged. Then mice were light adapted for 10 minutes at an intensity of $30 \mathrm{~cd}-\mathrm{s} / \mathrm{m}^{2}$ before photopic ERG measurements were recorded at stimulus intensity of $1.4 \mathrm{log} \mathrm{cd}-\mathrm{s} / \mathrm{m}^{2}$ in the presence of continuous 30 Ganzfeld cd-s $/ \mathrm{m}^{2}$ background light with inter-stimulus intervals of 0.4 seconds. Fifty recordings were averaged for each light-adapted ERGs measurement. B-wave amplitudes were defined as the difference between the trough and peak of each waveform. Scotopic and photopic b-wave amplitudes were averaged presented as mean \pm SD.

\section{Statistical analysis}

Statistical analysis was performed with unpaired t-test and significance defined as a $\mathrm{P}$ value of less than 0.05 .

\section{Results}

Wild type mice injected with the AAV5-smCBA-mCherry vector in the eyes were euthanized 2 weeks post-injection. Retinal whole mount and frozen sectioning were performed to characterize mCherry expression. The 8 whole mounts of the wild-type mouse eyes injected with $0.5 \mu \mathrm{L}$ of vector (Figure $1 \mathrm{~A}$ ) and the 8 whole mounts of the eyes injected with $1 \mu \mathrm{L}$ of vector (Figure $1 \mathrm{~B}$ ) showed wide spread mCherry expression across the entire retina. The average diameters of retinal whole mounts in the two groups were similar for the right and left eyes, $4670 \pm 121 \mu \mathrm{m}$ and $4676 \pm 145 \mu \mathrm{m}$, respectively. Consistent with whole mount, frozen sections from 2 wild-type mice (Figure 1C and 1D) also showed mCherry positive staining from the optic nerve to the margin of the retina with both injection volumes. Frozen sections showed that the eyes injected with $0.5 \mu \mathrm{L}$ of vector presented a same florescence with the eyes injected with $1 \mu \mathrm{L}$ of vector.

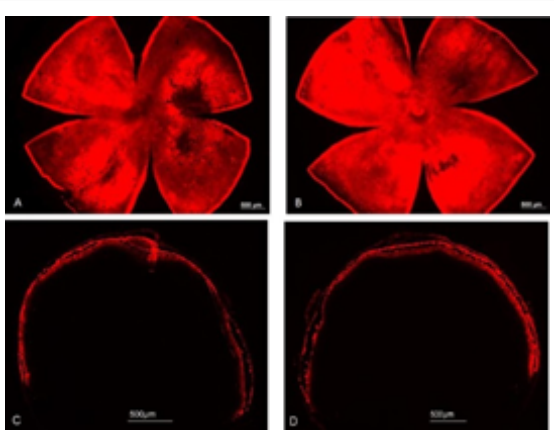

Figure 1: Red mCherry expression in retinal whole mounts and frozen section two weeks after sub retinal injection of the AAV5-smCBA-mCherry vector in P14 C57 BL/6J mice Red fluorescence in retinal whole mounts ( $A$ and $B$ ) and frozen sections $(C$ and $D)$ at low magnification. ( $A$ and $C$ ): $0.5-\mu L-v e c t o r$ injection; ( $B$ and $D): 1.0-\mu L$-vector injection. Exposure and gain settings were the same across all images.

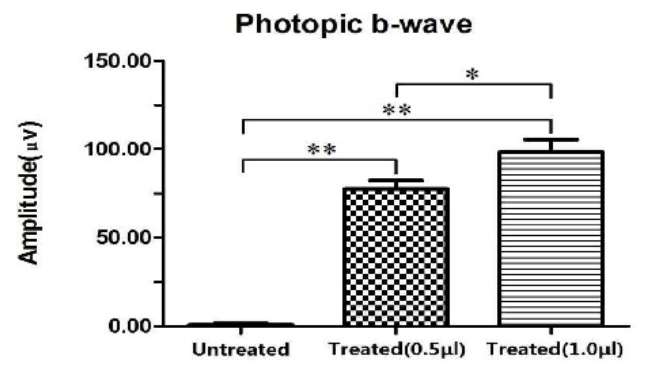

Figure 2: Light-adapted cone-derived ERG amplitudes from Cngb3 KO mouse eyes 2 months after subretinal injection of $0.5 \mu \mathrm{L}$ and $1 \mu \mathrm{L}$ of AAV5-IRBP/GNAT2hCngb3 vector Comparison of average values for photopic contrast sensitivities; NS: no statistical difference, ${ }^{*}: \mathrm{P}<0.05,{ }^{* \star}: \mathrm{P}<0.0001$.

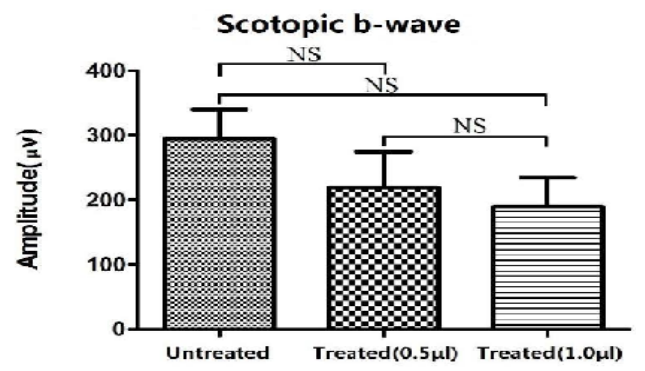

Figure 3: Dark-adapted rod-derived ERG b-wave amplitudes from the eyes of Cngb3 KO mice 2 months after subretinal injection of $0.5 \mu \mathrm{L}$ and $1 \mu \mathrm{L}$ of AAV5IRBP/GNAT2-hCngb3 vector. Although there was a reduction of the average value for scotopic ERG b-wave amplitude, there was no statistically significant difference between these three groups. NS: no statistical difference.

Light-adapted cone-derived ERG responses were recorded in both eyes from 8 of the $10 \mathrm{Cngb3} \mathrm{KO}$ mice 2 months after AAV5-IRBP/ GNAT2-hCngb3 treatment (Figure 2). The photopic b-wave amplitudes average was $77.5 \pm 13.887 \mu \mathrm{V}$ for the $0.5 \mu \mathrm{L}$ injected right eyes and that of the $1-\mu \mathrm{L}$-injected left eyes was $98.125 \pm 21.537 \mu \mathrm{V}$. The ERG response from the eyes of untreated mice was $1.25 \pm 2.5 \mu \mathrm{V}$. The 0.5 $\mu \mathrm{L}$ and $1-\mu \mathrm{L}$-vector injected eyes showed statistically higher responses than those from the eyes of untreated control mice $(\mathrm{P}<0.0001)$. We also found statistical difference between the $0.5-\mu \mathrm{L}$ - and $1-\mu \mathrm{L}$-vectortreated eyes $(\mathrm{P}=0.035)$.

In both experimental conditions, the eyes showed normal rodderived ERG waveforms after 2 months of treatment (Figure 3). The average scotopic b-wave amplitude of the $0.5 \mu \mathrm{L}$ injected right eyes was 
$220 \pm 109.545 \mu \mathrm{V}$ and that of the $1-\mu \mathrm{L}$-injected left eyes was $190 \pm 88.694$ $\mu \mathrm{V}(\mathrm{P}=0.685)$. The average $\mathrm{b}$-wave amplitude measure in the eyes of untreated mice was $295 \pm 90 \mu \mathrm{V}$. Although there was a reduction of the scotopic ERG b-wave amplitude, there was no statistically significant difference between these three groups $(\mathrm{P}>0.05)$.

\section{Discussion}

The subretinal space is an excellent target for gene therapy $[6,8$ $13]$ and drug delivery $[18,19]$ for retinal diseases. In fact, subretinally injected vectors get directly in contact with photoreceptors and RPE cells. However, the large area of retinal detachment induced by subretinal injection can cause cone death in the fovea area. In a 3-year study [12], 3 of 4 patients, experiencing detached fovea during surgery, lost fovea thickness, presumably due to fovea cone loss, as assessed by follow-up optical coherence tomography (OCT) analysis.

Our previous study using a LCA2 mouse model clearly demonstrated a dose-response relationship between the dose of vector and a better rescue [17]. Thus, in order to get the best results from gene therapy in knockout mouse models, vectors should be injected into the subretinal space. From our experience in mouse subretinal injection, a vector volume of $1 \mu \mathrm{L}$ is optimal to achieve the entire retinal detachment $[11,20-29]$. When $1.0 \mu \mathrm{L}$ of vector is successfully injected into the mouse subretinal space, usually three blebs form underneath the retina, which indicates a good injection. The blebs induced by the injection normally regress within $24 \mathrm{hrs}$. In this study, when $0.5 \mu \mathrm{L}$ of vector was administered, only one or two blebs formed and only parts of the retina detached at the time of injection. However, fundus images of retinal whole mounts indicated that red fluorescence was distributed throughout the whole retina 2-month following the injection of both 0.5 $\mu \mathrm{L}$ and $1 \mu \mathrm{L}$ of vector, perhaps due to a diffusion effect after injection. There was no difference in the diameter of the vector expression areas between the two groups two weeks after injection. Therefore, $0.5 \mu \mathrm{L}$ of AAV5-smCBA-mCherry vector could also successfully induce the full detachment of the retina in mice.

Sustained long-term gene therapy rescue has been demonstrated in the Cngb3 KO mouse model [30,31]. Light-adapted cone-derived ERG was rescued for at least 6 months by subretinal injection of 1 $\mu \mathrm{L}$ of a human Cngb3 cDNA driven by a cone-targeting promoter IRBP/GNAT2 packaged into the AAV serotype 5 (AAV5-IRBP/ GNAT2-hCngb3, 10e10vg/ $\mu \mathrm{L}$ ) vector at P14 [31]. In this study, when compared to the $0.5 \mu \mathrm{L}$ injection, the $1.0-\mu \mathrm{L}$ injection increased ERG reduction, although there was no statistical significance. However, the $1 \mu \mathrm{L}$ injection induced a more robust cone-driven ERG response than the $0.5 \mu \mathrm{L}$ injection, indicating that the restoration of cone function is strongly correlated with the AAV dose/particles in the conditions used in this study. Therefore, reducing vector volume may only work in Cngb3 KO mice when the vector titer is very high. Our finding showing that the $0.5 \mu \mathrm{L}$ injection can successfully induce the detachment of the entire retina would be helpful in treatments where toxicity is an issue. Toxicities caused by gene overexpression have been reported in various scenarios [32]. Thus, a vector volume of $0.5 \mu \mathrm{L}$ vector in combination with the proper vector titer may provide a safe and effective treatment strategy in gene therapies when toxicity is a concern.

\section{Conclusion}

Our data showed that reduced volume $(0.5 \mu \mathrm{L})$ of vector induced fewer damages. However, higher doses of vector $(1 \mu \mathrm{L})$ restore higher ERG function in Cngb3 KO mouse.

\section{Acknowledgments}

This work was supported by NIH Grant EY023543, EY021721, and EY018331, by foundation fighting blindness, BCM family foundation, Research to prevent blindness, Inc. the National Natural Science Foundation of China (81371060 and $81260155)$, a retinal gene therapy study grant from Wenzhou Medical University Wenzhou, China (QTJ11018), and the Jiangsu Province Foundation for Innovative Research Team.

\section{References}

1. Daiger SP, Bowne SJ, Sullivan LS (2007) Perspective on genes and mutations causing retinitis pigmentosa. Arch Ophthalmol 125: 151-158.

2. Heckenlively JR, Daiger SP (2007) Hereditary retinal and choroida degenerations, in Emery and Rimoin's Principals and practices of medical genetics. (5thedn) Philadelphia: Churchill Livingston Elsevier.

3. Berger W, Kloeckener-Gruissem B, Neidhardt J (2010) The molecular basis of human retinal and vitreoretinal diseases. Prog Retin Eye Res 29: 335-375.

4. http://www.sph.uth.tmc.edu/RetNet/.

5. Acland GM, Aguirre GD, Ray J, Zhang Q, Aleman TS, et al. (2001) Gene therapy restores vision in a canine model of childhood blindness. Nat Genet 28: 92-95.

6. Bennicelli J, Wright JF, Komaromy A, Jacobs JB, Hauck B, et al. (2008) Reversal of blindness in animal models of leber congenital amaurosis using optimized AAV2-mediated gene transfer. Mol Ther 16: 458-465.

7. Pang JJ, Chang B, Hawes NL, Hurd RE, Davisson MT, et al. (2005) Retina degeneration 12 (rd12): a new spontaneously arising mouse model for human Leber congenital amaurosis (LCA). Mol Vis 11: 152-162.

8. Pang JJ, Chang B, Kumar A, Nusinowitz S, Noorwez SM, et al. (2006) Gene therapy restores vision-dependent behavior as well as retinal structure and function in a mouse model of RPE65 Leber congenital amaurosis. Mol Ther 13: $565-572$.

9. Cideciyan AV, Hauswirth WW, Aleman TS, Kaushal S, Schwartz SB, et al. (2009) Human RPE65 gene therapy for Leber congenital amaurosis: persistence of early visual improvements and safety at 1 year. Hum Gene Ther 20: 999-1004

10. Deng WT, Dinculescu A, Li Q, Boye SL, Li J, et al. (2012) Tyrosinemutant AAV8 delivery of human MERTK provides long-term retinal preservation in RCS rats. Invest Ophthalmol Vis Sci 53: 1895-1904.

11. Christiana JJ, Lennart B, Micah AC, Redmond TM, Jeffrey HB, et al. (2008) Technical Brief: Subretinal injection and electroporation into adult mouse eyes. Mol Vis 14: 2211-2226.

12. Jacobson SG, Cideciyan AV, Ratnakaram R, Heon E, Schwartz SB, et al (2012) Gene therapy for leber congenital amaurosis caused by RPE65 mutations: safety and efficacy in 15 children and adults followed up to 3 years. Arch Ophthalmol 130: 9-24.

13. Jacobson SG, Acland GM, Aguirre GD, Aleman TS, Schwartz SB, et al. (2006) Safety of recombinant adeno-associated virus type 2-RPE65 vector delivered by ocular subretinal injection. Mol Ther 13: 1074-1084.

14. Kohl S, Varsanyi B, Antunes GA, Baumann B, Hoyng CB, et al. (2005) CNGB3 mutations account for $50 \%$ of all cases with autosomal recessive achromatopsia. Eur J Hum Genet 13: 302-308.

15. Chiu MI, Nathans J (1994) Blue cones and cone bipolar cells share transcriptional specificity as determined by expression of human blue visual pigment-derived transgenes. J Neurosci 14: 3426-3436.

16. Yang GS, Schmidt M, Yan Z, Lindbloom JD, Harding TC, et al. (2002) Virusmediated transduction of murine retina with adeno-associated virus: effects of viral capsid and genome size. J Virol 76: 7651-7660.

17. Timmers AM, Zhang H, Squitieri A, Gonzalez-Pola C (2001) Subretinal injections in rodent eyes: effects on electrophysiology and histology of rat retina. Mol Vision 7: 131-137.

18. Maia M, Kellner L, de Juan E Jr, Smith R, Farah ME, et al. (2004) Effects of indocyanine green injection on the retinal surface and into the subretinal space in rabbits. Retina 24: 80-91.

19. Shen WY, Rakoczy PE (2001) Uptake dynamics and retinal tolerance of phosphorothioate oligonucleotide and its direct delivery into the site of choroidal neovascularization through subretinal administration in the rat. Antisense Nucleic Acid Drug Dev 11: 257-264.

20. Roman AJ, Boye SL, Aleman TS, Pang JJ, McDowell JH, et al. (2007) Electroretinographic analyses of Rpe65-mutant rd12 mice: developing an in 
Citation: Liu X, Zhang Y, Du W, Shi W, Tao Y, et al. (2016) Evaluation of AAV-Mediated Gene Therapy with Reduced Vector Volume in Cngb3 Knockout Mice, a Model of Achromatopsia. Hereditary Genet 5: 163. doi:10.4172/2161-1041.1000163

vivo bioassay for human gene therapy trials of Leber congenital amaurosis. Mol Vis 13: 1701-1710.

21. Bemelmans AP, Kostic C, Crippa SV, Hauswirth WW, Lem J, et al. (2006) Lentiviral gene transfer of RPE65 rescues survival and function of cones in a mouse model of Leber congenital amaurosis. PLoS Med 3: e347.

22. Pang JJ, Boye SE, Lei B, Boye SL, Everhart D, et al. (2010) Selfcomplementary AAV-mediated gene therapy restores cone function and prevents cone degeneration in two models of Rpe65 deficiency. Gene Ther 17: 815-826.

23. Li X, Li W, Dai X, Kong F, Zheng Q, et al. (2011) Gene therapy rescues cone structure and function in the 3-month-old rd12 mouse: a model for midcourse RPE65 leber congenital amaurosis. Invest Ophthalmol Vis Sci 52: 7-15.

24. Vollrath D, Feng W, Duncan JL, Yasumura D, D'Cruz PM, et al. (2001) Correction of the retinal dystrophy phenotype of the RCS rat by viral gene transfer of Mertk. Proc Natl Acad Sci USA 98: 12584-12589.

25. Smith AJ, Schlichtenbrede FC, Tschernutter M, Bainbridge JW, Thrasher AJ (2003) AAV-Mediated gene transfer slows photoreceptor loss in the RCS rat model of retinitis pigmentosa. Mol Ther 8: 188-195.

26. Kong J, Kim SR, Binley K, Pata I, Doi K, et al. (2008) Correction of the disease phenotype in the mouse model of Stargardt disease by lentiviral gene therapy. Gene Ther 15: 1311-1320.
27. Han Z, Conley SM, Makkia RS, Cooper MJ, Naash MI (2012) DNA nanoparticlemediated ABCA4 delivery rescues Stargardt dystrophy in mice. J Clin Invest 122: $3221-3226$

28. Boye SE, Boye SL, Pang J, Ryals R, Everhart D, et al. (2010) Functional and behavioral restoration of vision by gene therapy in the guanylate cyclase-1 (GC1) knockout mouse. PLoS ONE 5: e11306.

29. Boye SL, Conlon T, Erger K, Ryals R, Neeley A, et al. (2011) Long-term preservation of cone photoreceptors and restoration of cone function by gene therapy in the guanylate cyclase-1 knockout (GC1KO) mouse. Invest Ophthalmol Vis Sci 52: 7098-7108.

30. Carvalho LS, Xu J, Pearson RA, Smith AJ, Bainbridge JW, et al. (2011) Longterm and age-dependent restoration of visual function in a mouse model of CNGB3-associated achromatopsia following gene therapy. Hum Mol Genet 20: 3161-3175.

31. Shi W, Mao S, Deng WT, Li J, Liu X, et al. (2012) Cone Targeted AAV-mediated Gene Therapy Restores Cone Function in the Cngb3 Knockout Mouse, a Mode of Human Achromatopsia 1. Invest Ophthalmol Vis Sci 53: E-Abstract 1903.

32. Seo SJ, Robert FM, Alina VD, Sajag B, Daniel G, et al. (2013) Subretinal Gene Therapy of Mice With Bardet-Biedl Syndrome Type 1. Invest Ophthalmol Vis Sci 54: 6118-6132. 